\title{
Homogenization of dislocation dynamics
}

\author{
Ahmad El Hajj, Hassan Ibrahim and Régis Monneau \\ CERMICS, ENPC, 6 \& 8 avenue Blaise Pascal, Cité Descartes, Champs sur Marne, 77455 \\ Marne-la-Vallée Cedex 2, France \\ E-mail: elhajj@cermics.enpc.fr, ibrahim@cermics.enpc.fr, monneau@cermics.enpc.fr
}

\begin{abstract}
In this paper we consider the dynamics of dislocations with the same Burgers vector, contained in the same glide plane, and moving in a material with periodic obstacles. We study two cases: i) the particular case of parallel straight dislocations and ii) the general case of curved dislocations. In each case, we perform rigorously the homogenization of the dynamics and predict the corresponding effective macroscopic elasto-visco-plastic flow rule.
\end{abstract}

\section{Introduction}

In the recent years, an important effort has been done, both to improve the methods to compute discrete dislocation dynamics (see for instance the book of Bulatov and Cai [1] and the references therein) and also to connect them to continuum models of plasticity in crystalline solids (see for instance Fivel et al. [2] and more recently Hoc et al. [3]). Although continuum models of dislocations are known since the 50's (see Kröner $[4,5]$ ), the dynamics has been taken into account only recently : see Groma et al. [6,7] in 2D (and their mathematical studies in [8,9]), Hochrainer et al. [10], and Monneau [11] in 3D. The goal of our work is to present, on a particular example, a rigorous justification of a continuum model with densities of dislocations bridging the gap with dislocation dynamics at the microscale. Indeed for a very special geometry, we are able to deduce by homogenization, the macroscopic elasto-visco-plastic flow rule relating the plastic strain velocity to the shear stress. The full technical details are presented in [12].

\section{Homogenization of straight dislocations}

In this section, we consider the case of parallel straight edge dislocations with the same Burgers vector $\mathbf{b}=b e_{x}$ with $b>0$, where $\left(e_{x}, e_{y}, e_{z}\right)$ is an orthonormal basis with corresponding coordinates $(x, y, z)$. All these dislocation lines are assumed to be contained in the same glide plane $(x, y)$ and to move in this plane.

\subsection{The microscopic model for straight dislocations}

Because of our assumptions, for every integer $i \in \mathbb{Z}$, we can simply describe the position of the $i$-th dislocation by its real abscissa that we call $x_{i}(t)$ where $t$ is the time. We want to take into account the interactions of each dislocation with other defects in the crystal, that constitute obstacles to their motion. Those obstacles can be for instance other pinned dislocations or precipitates. In order to simplify the analysis, we will assume that these obstacles are periodically distributed, of spatial period $\lambda$. In our model, those obstacles will be simply modeled by a smooth periodic potential $V^{\text {per }}$ satisfying $V^{\text {per }}(x+\lambda)=V^{\text {per }}(x)$. Then the energy of the system is the 
sum of two contributions: the interactions of each dislocations with the periodic potential and the sum of the two-body interactions between dislocations associated to a pair potential $V$. The energy of a set of dislocations is then given by

$$
E=\sum_{i} V^{p e r}\left(x_{i}\right)+\sum_{i<j} V\left(x_{i}-x_{j}\right) \quad \text { with } \quad V(x)=-\bar{\mu} b \ln |x| \quad \text { and } \quad \bar{\mu}=\frac{\mu}{2 \pi(1-\nu)}
$$

where the constants $\mu$ and $\nu$ are respectively the shear modulus and the Poisson ratio. Remark that the force $-V^{\prime}(x)$ is then the usual Peach-Koehler force created at the point $x$ by an edge dislocation positioned at the origin.

We then consider the fully overdamped dynamics, where the velocity is proportional to the force, i.e.

$$
B \frac{d x_{i}}{d t}=-\nabla_{x_{i}} E+\tau_{e x t}
$$

where $B$ is the viscous drag coefficient and the force is on the right hand side. The first contribution to the force is a term deriving from the energy and $\tau_{\text {ext }}$ is a real exterior applied shear stress, that can be seen as a driving force of the system. A natural question is then: what is the macroscopic behavior of this system?

In order to answer this question (which is done in Theorem 2.1), we have to introduce the plastic strain. To each dislocation is associated a three-dimensional displacement in the crystal, whose plastic strain is localized in the glide plane $z=0$ and is equal to $\gamma \delta_{0}(z)$ where $\delta_{0}$ is the Dirac mass. For instance, for a dislocation $x_{i}$, the intensity $\gamma$ (that we continue to call plastic strain) is equal to $-b H\left(x-x_{i}\right)$ where the Heaviside function $H(x)$ is equal to 1 for positive $x$ and zero otherwise. Here the sign defining the plastic strain is such that the quantity $\gamma$ increases when $x_{i}$ increases. Then the total plastic strain can be written as

$$
\gamma(x, t)=-b \sum_{i} H\left(x-x_{i}(t)\right)
$$

\subsection{The normalization procedure}

We are now interested in the behavior of the system at a macroscopic scale $\Lambda$ such that $\Lambda>>\lambda=\bar{\lambda} b$ where $\bar{\lambda}>1$ is a fixed ratio. Then we introduce several dimensionless quantities. We call $\bar{x}$ and $\bar{t}$ the normalized spatial and time coordinates at the macroscopic level, and introduce a parameter $\varepsilon$ and the associated normalized macroscopic plastic strain $\gamma^{\varepsilon}$ such that

$$
\bar{x}=\frac{x}{\Lambda}, \quad \bar{t}=\frac{\bar{\mu}}{B} \frac{t}{\Lambda}, \quad \varepsilon=\frac{b}{\Lambda} \quad \text { and } \quad \gamma^{\varepsilon}(\bar{x}, \bar{t})=\frac{1}{\Lambda} \gamma(x, t) \quad \text { with } \quad \gamma^{\varepsilon}(\bar{x}, 0)=\varepsilon\left[\frac{1}{\varepsilon} \gamma_{0}(\bar{x})\right]
$$

where [.] is the floor function, $\gamma_{0}$ is a given function and $B \Lambda / \bar{\mu}$ is a typical macroscopic time deduced from equation (2.1). Remark that $\varepsilon$ can be very small in our application (for instance $\varepsilon \simeq 10^{-6}$ if $b \simeq 10^{-9} \mathrm{~m}$ and $\Lambda \simeq 10^{-3} \mathrm{~m}$ ).

We expect that the macroscopic behavior of the model is well described by the limit macroscopic plastic strain $\gamma^{0}(\bar{x}, \bar{t})$ of $\gamma^{\varepsilon}(\bar{x}, \bar{t})$ as $\varepsilon$ goes to zero.

\subsection{Heuristics for the macroscopic stress field}

In this subsection, we want to give heuristic expressions of the normalized dislocation density and the macroscopic stress field, in terms of the limit macroscopic plastic strain.

We remark that the gradient of the map $x \mapsto-\gamma^{\varepsilon}(x / \Lambda, \bar{t}) / \varepsilon$ is a sum of Dirac masses, and then the number of dislocations in a large segment of length $\Delta x$ is formally given by $-\int_{0}^{\Delta x} \frac{1}{\varepsilon \Lambda} \frac{\partial \gamma^{\varepsilon}}{\partial \bar{x}}(x / \Lambda, \bar{t}) d x$. This shows at least formally that the dislocation density can be 
estimated as $\rho(x, t)=-\frac{1}{\varepsilon \Lambda} \frac{\partial \gamma^{0}}{\partial \bar{x}}(\bar{x}, \bar{t})$. Then the total stress on the right hand side of $(2.1)$ can be formally described at the macroscopic scale by

$$
\tau=\tau_{e x t}+\tau_{s c} \quad \text { with } \quad \tau_{s c}(\bar{x}, \bar{t})=-\bar{\mu} \int_{-\infty}^{+\infty} \frac{d \bar{x}^{\prime}}{\bar{x}-\bar{x}^{\prime}} \frac{\partial \gamma^{0}}{\partial \bar{x}}\left(\bar{x}^{\prime}, \bar{t}\right)
$$

where we take the principal value in the integral defining the self-consistent field $\tau_{s c}$. This expression can be deduced from the equation $\tau_{s c}(\bar{x}, \bar{t})=-\left(V^{\prime} \star_{x} \rho\right)(x, t)$, where $\star_{x}$ denotes the convolution with respect to the variable $x$. Remark also that the expression (2.3) of $\tau_{s c}$ is known to be the resolved shear stress created by the normalized dislocation density

$$
\rho^{0}=-\frac{\partial \gamma^{0}}{\partial \bar{x}}
$$

where for instance $\rho^{0}=1 / \bar{\lambda}$ when there is one dislocation by spatial period $\lambda$. In particular, we see that $\tau_{s c}$ keeps the memory of the long range interactions between dislocations.

\subsection{The homogenization result}

We expect that the effective equation satisfied by the limit $\gamma^{0}$ can be written

$$
\begin{cases}\frac{\partial \gamma^{0}}{\partial \bar{t}}=f\left(\rho^{0}, \tau\right), & \text { for all } \quad \bar{x} \in \mathbb{R}, \quad \bar{t} \in(0,+\infty), \\ \gamma^{0}(\bar{x}, 0)=\gamma_{0}(\bar{x}) \quad \text { for all } \quad \bar{x} \in \mathbb{R}\end{cases}
$$

where $\rho^{0}$ is given in (2.4) and $\tau$ in (2.3). Then our main result is:

\section{Theorem 2.1 (Homogenization of straight dislocations)}

Assume that the initial data $\gamma_{0}$ is non-decreasing and satisfies $\left|\gamma_{0}\right|+\left|\gamma_{0}^{\prime}\right|+\left|\gamma_{0}^{\prime \prime}\right| \leq C$ for some constant $C$. Then for any $C^{2}$ periodic potential $V^{\text {per }}$, there exists a continuous function $f: \mathbb{R}^{2} \rightarrow \mathbb{R}$ such that $\tau \mapsto f\left(\rho^{0}, \tau\right)$ is nondecreasing. And there exists a unique viscosity solution $\gamma^{0}$ of the equation (2.5).

Moreover, under the assumptions and notation of this section, there exists a unique solution $\gamma^{\varepsilon}$ associated to the dynamics (2.1) with initial data given in (2.2), and $\gamma^{\varepsilon}$ converges to $\gamma^{0}$ locally uniformly on $\mathbb{R} \times[0,+\infty)$.

This result is proven rigorously in [12] in the mathematical framework of viscosity solutions (see for instance Crandall, Ishii, Lions [13] for an introduction to this theory). We explain in the next section how we compute the function $f$, which keeps the memory of the short range interactions between the dislocations and the periodic potential $V^{\text {per }}$.

\subsection{Computation of $f$ using Orowan's law}

In this subsection, we briefly explain (without any justifications) how to compute the function $f$. We refer the reader to [12] for the proofs of those results.

Case $A: V^{\text {per }} \equiv 0$.

In this special case, we can show that

$$
f\left(\rho^{0}, \tau\right)=\rho^{0} \bar{v} \quad \text { with } \quad \bar{v}=\frac{\tau}{\bar{\mu}}
$$

which is nothing else than the normalized Orowan's law giving, in a dimensionless form, the plastic strain velocity as the product of the normalized dislocation density $\rho^{0}$ and the normalized mean velocity $\bar{v}$ of the dislocations. 
Case B: General periodic potential $V^{\text {per }}$.

In that case, the function $f$ can be computed using the following two steps.

Step 1.

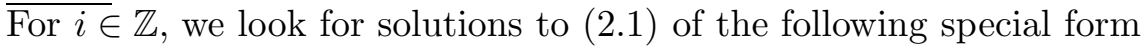

$$
x_{i}(t)=b \cdot h\left(\frac{v t}{b}+\frac{i}{\rho^{0}}\right), \quad \text { with } \quad h(a+\bar{\lambda})=\bar{\lambda}+h(a) \quad \text { for all } \quad a \in \mathbb{R}
$$

for some constant $v$ and for a function $h$ which is called a hull function. Both $v$ and $h$ have to be determined. Because of the convexity of the two-body potential $V$ outside the origin, it is possible to show that the constant $v$ exists and is unique. Moreover this constant $v$ can be interpreted as the mean velocity of each dislocation.

Step 2.

We simply define $f\left(\rho^{0}, \tau_{\text {ext }}\right)$ using the normalized Orowan's law as in (2.6), but with the normalized velocity $\bar{v}$ replaced by the constant $\bar{v}=\frac{B}{\bar{\mu}} v$.

\subsection{Numerical computation of $f$}

We present numerical simulations for the computation of the function $f$. We work with dimensionless quantities: $\lambda=1=\bar{\lambda}=b=B=\bar{\mu}$. We put initially $N$ dislocations in an interval of length $l=10$ which is repeated periodically. Therefore this interval contains $l$ times the period of the periodic potential that we choose equal to $V^{p e r}(x)=\frac{A}{2 \pi} \sin (2 \pi x)$ with $A=3$. We discretize the ODE system (2.1), using an explicit Euler scheme with a time step $\Delta t=0.01$. We compute numerically the mean velocity $v$ of the dislocations after a final time $T=1000$. We then set $f=\rho^{0} v$ with $\rho^{0}=N / l$. We do the computation with $N=1, \ldots, 200$ and $0 \leq \tau_{\text {ext }} \leq 9$ with $\Delta \tau_{\text {ext }}=\frac{9}{200}$. Remark that we can restrict our computation for positive $\tau_{\text {ext }}$, because we have $f\left(\rho^{0},-\tau_{\text {ext }}\right)=-f\left(\rho^{0}, \tau_{\text {ext }}\right)$, from the symmetry of the potential $V^{\text {per }}$ in our problem. The level sets of the function $f$ are represented on Figure 1. In order to have a better view of the set where $f=0$, this set is conventionally represented in Figure 1 with artificial negative values of $f$. We remark that this figure shows in particular a collective behavior of the dislocations: higher is the density of dislocations, then easier the dislocations move above the obstacles.

Figure 2 shows the map $\tau_{\text {ext }} \mapsto f\left(\rho^{0}, \tau_{\text {ext }}\right)$ for $\rho^{0}=N / l$ with $N=1,10,20$. We see in particular that for $\tau_{\text {ext }}$ under a threshold (that depends on the dislocation density $\rho^{0}$ ) the function $f$ vanishes.

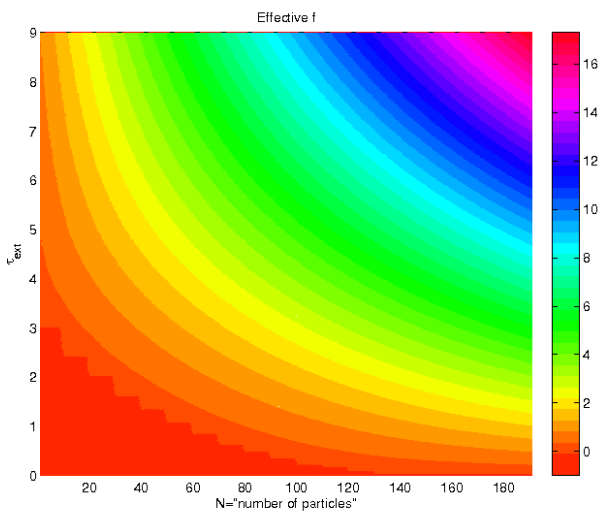

Figure 1. Level sets of the effective $f\left(N / l, \tau_{\text {ext }}\right)$ with $N$ on abscissas and $\tau_{\text {ext }}$ on ordinates

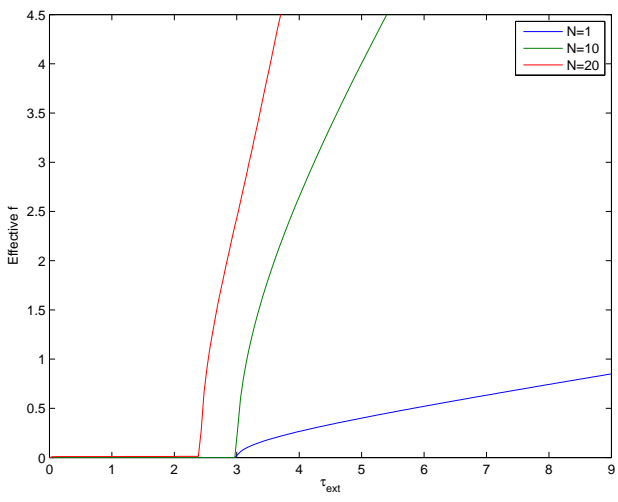

Figure 2. For $N=1,10,20$, graph of the map $\tau_{\text {ext }} \mapsto f\left(N / l, \tau_{\text {ext }}\right)$ 


\section{Homogenization of curved dislocations}

In this section, we very briefly generalize the previous analysis to the case of curved dislocations all contained in the same plane $(x, y)$ with the same Burgers vector $\mathbf{b}=b e_{x}$ with $b>0$.

\subsection{The microscopic model for curved dislocations}

For $i \in \mathbb{Z}$, the motion of the $i$-th dislocation curve $\Gamma_{i}(t)$ at the point $X \in \mathbb{R}^{2}$ is given by its normal velocity $\mathcal{V}$ defined by

$$
B \cdot \mathcal{V}(X, t)=\tau^{p e r}(X)+\sum_{j} F_{j}(X, t)
$$

where $F_{j}(X, t)$ is the resolved Peach-Koehler force created by the dislocation $\Gamma_{j}(t)$ at the point $X$. Here $\tau^{\text {per }}$ is a smooth periodic function satisfying $\tau^{\text {per }}(X+\lambda k)=\tau^{\text {per }}(X)$ for all $k \in \mathbb{Z}^{2}$, which represents the periodic obstacles to the motion of the dislocations and can also include the exterior applied stress. To give the expression of this force, it is convenient to introduce a continuous function $\tilde{\gamma}(X, t)$ such that each dislocation curve $\Gamma_{j}(t)$ can be seen as the level set $\tilde{\gamma}(X, t)=j b$ (when this level set is non-degenerated). Then a good approximation is given by

$$
F_{j}(X, t)=\frac{1}{2} \int_{\mathbb{R}^{2}} d Z J(X-Z) \operatorname{sign}(\tilde{\gamma}(Z, t)-j b)
$$

where, in the integral, the sign function takes values $-1,0,1$. Here the kernel $J$ is smooth and satisfies for a cut-off radius $R=\bar{R} b$ with $\bar{R}>1$ fixed:

$$
J(-X)=J(X) \geq 0, \quad \text { and } \quad J(X)=J_{\infty}(X):=\frac{1}{|X|^{3}} g\left(\frac{X}{|X|}\right) \quad \text { for } \quad|X|>R>0
$$

where for isotropic elasticity with $X=(x, y)$, we have $g\left(\frac{X}{|X|}\right)=\frac{\mu b}{4 \pi}\left\{\frac{x^{2}(2 \beta-1)+y^{2}(2-\beta)}{x^{2}+y^{2}}\right\}$ with $\beta=\frac{1}{1-\nu}$. Remark that this formula allows to describe with the same formalism edge, screw and mixed dislocations (see for instance [14]). We also define the plastic strain $\gamma$ as

$$
\gamma=b\left[\frac{\tilde{\gamma}}{b}\right]
$$

where we recall that [.] is the floor function. Then we proceed as in the previous section and define

$$
\bar{X}=\frac{X}{\Lambda}, \quad \bar{t}=\frac{\mu}{B} \frac{t}{\Lambda}, \quad \varepsilon=\frac{b}{\Lambda}, \quad \text { and } \quad \gamma^{\varepsilon}(\bar{X}, \bar{t})=\frac{1}{\Lambda} \gamma(X, t), \quad \text { with } \quad \gamma^{\varepsilon}(\bar{X}, 0)=\varepsilon\left[\frac{1}{\varepsilon} \gamma_{0}(\bar{X})\right] .
$$

\subsection{The homogenization result}

We expect that the effective equation satisfied by the limit $\gamma^{0}$ of $\gamma^{\varepsilon}$ can be written

$$
\left\{\begin{array}{l}
\frac{\partial \gamma^{0}}{\partial \bar{t}}=f\left(-\nabla \gamma^{0}, \tau_{s c}\right), \quad \text { for all } \quad \bar{X} \in \mathbb{R}^{2}, \quad \bar{t} \in(0,+\infty), \\
\gamma^{0}(\bar{X}, 0)=\gamma_{0}(\bar{X}) \quad \text { for all } \quad \bar{X} \in \mathbb{R}^{2}
\end{array}\right.
$$

with

$$
\tau_{s c}(\bar{X}, \bar{t})=\int_{\mathbb{R}^{2}} d Z J_{\infty}(\bar{X}-Z) \gamma^{0}(Z, \bar{t})
$$

where we take the principal value of the integral. Remark that this expression of $\tau_{s c}$ is consistent with the one given in (2.3) in the special case where $\gamma^{0}(\bar{x}, \bar{y}, \bar{t})$ is independent of $\bar{y}$. Then we have 


\section{Theorem 3.1 (Homogenization of curved dislocations)}

Assume that the initial data satisfies $\left|\gamma_{0}\right|+\left|\nabla \gamma_{0}\right|+\left|D^{2} \gamma_{0}\right| \leq C$ for some constant $C$. Then for any $C^{2}$ periodic function $\tau^{\text {per }}$, there exists a continuous function $f: \mathbb{R}^{2} \times \mathbb{R} \rightarrow \mathbb{R}$ such that $\tau \mapsto f(\cdot, \tau)$ is nondecreasing. And there exists a unique viscosity solution $\gamma^{0}$ of the equation (3.3).

Moreover, under the assumptions and notation of this section, there exists a unique solution $\gamma^{\varepsilon}$ associated to the dynamics (3.1) with initial data given in (3.2), and $\gamma^{\varepsilon}$ converges to $\gamma^{0}$ locally uniformly on $\mathbb{R}^{2} \times[0,+\infty)$.

\section{Conclusion}

The main result of our work is the justification of the elasto-visco-plastic flow rule by the homogenization of the dynamics of dislocations with the same Burgers vector, moving in the same glide plane with periodic obstacles. Even if this geometry is very particular, this is, up to our knowledge, the first rigorous result in this direction. We also explained how to compute the flow rule, and presented numerical results. The proof of the homogenization for straight dislocations uses strongly the local convexity of the two-body potential $V$ (which is equivalent to the non-negativity of the kernel $J$ in the case of curved dislocations).

Remark that for the same dynamics, it is possible to find non-convex potentials $V$, for which there is no homogenization. For a general geometry, there is in general no hope to find any convexity argument to justify homogenization. On the contrary, it seems reasonable to think that homogenization could arise in general, if we assume moreover that the dynamics is modified by the addition of a small random noise. But this is still an open problem to investigate.

\section{Acknowledgements}

This work was supported by the contract ANR MICA (2006-2009).

\section{References}

[1] Bulatov V V and Cai W, Oxford University Press, (2006).

[2] Fivel M, Tabourot L, Rauch E and Canova G R, J. Phys. IV, 8 (1998), 151-158.

[3] Hoc T, Devincre B and Kubin L P, In C. et al. Gundlach, editor, Riso National Laboratory, Denmark (2004), 43-59.

[4] Kröner E, Erg. Angew. Math. 5 (1958), 1-179, Berlin: Springer.

[5] Kröner E, Int. J. Solids and Structures 38 (2001), 1115-1134.

[6] Groma I and Balogh P, Mat. Sci. Eng. A 234-236 (1997), 249-252.

[7] Groma I, Cikor F F and Zaiser M, Acta Mater. 51 (2003), 1271-1281.

[8] El Hajj A and Forcadel N, Math. Comp. 77 (2008), 789-812.

[9] Ibrahim H, Jazar M and Monneau R, C. R. Acad. Sci. Paris, Ser I 346 (2008) 945-950.

[10] Hochrainer T, Zaiser M and Gumbsch P, Philosophical Magazine 87 (8 \& 9) (2007), 1261-1282.

[11] R. Monneau, Interfaces Free Bound. 9 (2007), 383-409.

[12] Forcadel N, Imbert C and Monneau, Discrete Contin. Dyn. Syst. A 23 (3), to appear (March 2009), and HAL: hal-00140545 (12-27-2007).

[13] Crandall M G, Ishii H and Lions P -L, Bull. Amer. Math. Soc. (N.S.) 27 (1992), 1-67.

[14] Alvarez O, Hoch P, Le Bouar Y and Monneau R, Arch. Ration. Mech. Anal. 181 (3) (2006), 449-504. 